reported in two unrelated boys with consanguineous parents from the Centre de Génétique Médicale, Service de Pediatrie Générale and Radiologie, Hôpital d'Enfants de la Timone, Marseille, France. An autosomal recessive mode of inheritance is suggested and echographic survey of further pregnancies is advised. Clinical manifestations included macrocephaly, bulging forehead, antimongoloid slant of eyes, broad short nose, posteriorly rotated ears, herniae, polydactyly, cardiac defect, mental retardation and corpus callosal agenesis. (Philip $\mathrm{N}$ et al. The acrocallosal syndrome. Eur $\mathrm{J}$ Pediatr Feb $1988 ; 147: 206-208$ ).

COMrent. Only 12 cases of the syndrome have been reported. Schinzel, who described the syndrame in 1979 and has at least 5 publications on the subject, deserves the eponym.

\title{
FRAGILB-X SYNDRONE
}

A characteristic epileptogenic EEG pattern is described in five of 12 male subjects with fragile-X syndrome evaluated at the Instituto Oasi, via C. Ruggero, Troina, Italy, and Clinica Neurologica, II Universita Roma and Bologna, Italy. Focal paroxysmal temporal spikes, at times multifocal, occurred in sleep in one non-epileptic and four epileptic patients with mental retardation and fragile-X syndrome, but not in subjects with mental retardation, with or without epilepsy but without the fragile-X chromosome. (Musumeci SA et al. Fragile-X syndrome: A particular epileptogenic EEG pattern. Epilepsia Jan/Feb $1988 ; 29: 41-7)$.

Comenr. The authors believe that epilepsy must be considered an important clinical feature of fragile- $X$ syndrome, occurring in an average of $26 \%$ of reported cases. Karyotyping is advised in mentally retarded patients with epilepsy, even in those without typical clinical features or positive family history and especially in children who frequently lack the characteristic facial dysmorphisms and macro-orchidism (see Ped Neur Briefs $1987 ; \underline{1}: 41)$.

\section{INIRACRANIAL TUMORS}

\section{NEUROPIBROMATOSIS AND ACOUSTIC NEURONAS}

The criteria for diagnosis, treatment, family counseling and advances in genetics of neurofibromatosis are reviewed by a neurosurgeon and epidemiologist at the Massachusetts General Hospital, Boston, and the National Institute of Neurological Disorders, Bethesda, MD.

The neurofibromatoses consist of two distinct disorders, a peripheral and a central type, with genes located on separate chromosomes. The diagnosis of neurofibromatosis 1 (NFl, von Recklinghausen's neurofibromatosis or VRNF in Europe) requires two or more of the following: 6 or more cafe au lait macules, 2 or more neurofibromas, axillary or inguinal skin freckles, optic glioma, Lisch iris nodules, osseous lesion and familial occurrence. Neurofibromatosis 2 (NF2, bilateral acoustic neurofibromatosis or BANF in Europe) requires one of the following for diagnosis: a) bilateral eighth nerve tumors, or b) a positive family history plus a unilateral eighth nerve tumor or two of the following: neurofibroma, meningioma, glioma, Schwannoma, or lenticular opacity. For patients with NF2 there is a $50 \%$ risk of transmission to any offspring, and close relatives should be screened for cafe au lait spots or neurofibromas, acoustic nerve tumors and lens opacities. Acoustic neuromas commonly become symptomatic during or soon after puberty and 
may be exacerbated in pregnancy, suggesting a hormone or growth factor important in tumor formation. The inherited gene for NF2 is on the long arm of chromosone 22. (Martuza RL, Eldridge R. Neurofibromatosis 2 (Bilateral acoustic neurofibromatosis). N Engl J Med March 17 1988;318:684-8).

COMment. The British Neurofibromatosis Association -- LINK, and two voluntary support organizations in the US -- The National Neurofibromatosis Foundation and the Acoustic Neuroma Association, organize medical symposia and provide valuable assistance to patients and their families. Newer diagnostic methods including brain-stem auditory evoked potentials, CT and MRI and routine eye examination for subcapsular cataracts should afford earlier recognition.

Unidentified bright objects (UBO's), areas of increased signal on $\mathrm{Tl}$ or T2 weighted MRI, with concurrent negative contrast-enhanced CT are reported in 28 children with neurofibromatosis 1 and may possibly represent heterotopias. They were not correlated with the presence of learning disorders or retardation, and their significance is unclear. (Rubinstein $\mathrm{AE}$ et al. and Dunn DW, Roos KL. Neurology March 1988;38 (Suppl 1);282). I have a patient, a boy aged 12 yrs, with migraine headaches, macrocephaly, and cafe au lait patches, whose MRI shows UBO's. Although considered benign, such patients deserve careful long-term follow-up.

\section{INPANTILE SEIZURES}

\section{NEONATAL SEIZURES}

The clinical patterns, etiologies and times of onset of seizures were determined in a retrospective study of 150 newborns with seizures evaluated between 1982-87 in the neonatal intensive care unit at Washington University School of Medicine, Children's Hospital, St. Louis, MO. Subtle seizures in 97 (65\%) infants were characterized by eye movements, sucking, lip-smacking, chewing, tonic posturing, pedaling or rowing movements, and/or apneic spells. other seizure patterns were multifocal clonic (54\%), focal clonic, generalized tonic and myoclonic. Of $78 \%$ patients with at least one EEG, only $11 \%$ had ictal tracings. Hypoxicischemic encephalopathy (HIE) in $65 \%$ of the total was the most common cause of seizures in both preterm and term infants and $90 \%$ occurred in the first two days. Intracranial hemorrhage accounted for $10 \%$ of seizures. Other less common causes were metabolic, developmental defect, infection and drug withdrawal. In those with developmental defects, the onset of seizures was after 7 days of age. (Calciolari G, Perlman JM, Volpe JJ. Seizures in the neonatal intensive care unit of the 1980's. Clinical Pediatrics March 1988; 27:119-23).

COMranr. The authors admit that the absence of simultaneous video-EEG monitoring may lead to misinterpretation of the nature of some clinical phenomena regarded as seizures. Subtle seizures reported in more than half of the patients and associated with HIE have an inconsistent or no relationship to EEG seizure discharges (Mizrahi EM, Kellaway P. Neurology 1987;37:1837), and some authorities advise against anticonvulsant therapy unlesS EEG epileptiform confirmation is obtained (Ped Neur Briefs $1987 ; 1: 47-8)$. Self-resolution of "seizures" cannot be ruled out in a proportion of those apparently responding to anticonvulsant drugs, a therapy not without potential toxicity. 